Revue musicale OICRM

\title{
La migration numérique d'une oeuvre pionnière avec live electronics. Mesa (1966) de Gordon Mumma
}

\section{Jonathan Goldman, Francis Lecavalier et Ofer Pelz}

Volume 6, numéro 2, 2020

Musicologie numérique. Vers de nouvelles frontières disciplinaires

URI : https://id.erudit.org/iderudit/1068383ar

DOI : https://doi.org/10.7202/1068383ar

Aller au sommaire du numéro

Éditeur(s)

Observatoire interdisciplinaire de création et recherche en musique (OICRM)

ISSN

2368-7061 (numérique)

Découvrir la revue

Citer cet article

Goldman, J., Lecavalier, F. \& Pelz, O. (2020). La migration numérique d'une oeuvre pionnière avec live electronics. Mesa (1966) de Gordon Mumma. Revue musicale OICRM, 6(2), 1-24. https://doi.org/10.7202/1068383ar
Résumé de l'article

Cet article résume les retombées d'un projet de reconstruction d'une oeuvre pionnière de live electronics, à savoir Mesa (1966) du compositeur étatsunien Gordon Mumma (né en 1935). Une reconstruction de cette oeuvre a été présentée dans le cadre du festival Montréal/Nouvelles Musiques le 27 février 2017 par l'auteur et les collaborateurs de cet article : Jonathan Goldman au bandonéon, Francis Lecavalier à la conception informatique, et Ofer Pelz à l'interprétation de la partie électronique. Au cours de cette "migration ", l'oeuvre, fondamentalement analogique, a été reconçue pour un équipement numérique contemporain avec le consentement du compositeur. S'inscrivant nettement dans la lignée de l'École de New York cageienne, Mesa revêt un statut ontologique curieux : elle est le produit d'une indétermination passablement radicale. Comme c'est le cas pour l'oeuvre de Cage, Mesa et sa reconstruction numérique nous force à repenser l'ontologie et l'épistémologie de l'oeuvre musicale. 


\title{
La migration numérique d'une œuvre pionnière avec live electronics. Mesa (1966) de Gordon Mumma
}

\author{
Jonathan Goldman, avec le concours \\ de Francis Lecavalier et Ofer Pelz
}

\begin{abstract}
Résumé
Cet article résume les retombées d'un projet de reconstruction d'une œuvre pionnière de live electronics, à savoir Mesa (1966) du compositeur étatsunien Gordon Mumma (né en 1935). Une reconstruction de cette œuvre a été présentée dans le cadre du festival Montréal/Nouvelles Musiques le 27 février 2017 par l'auteur et les collaborateurs de cet article : Jonathan Goldman au bandonéon, Francis Lecavalier à la conception informatique, et Ofer Pelz à l'interprétation de la partie électronique. Au cours de cette " migration ", l'œuvre, fondamentalement analogique, a été reconçue pour un équipement numérique contemporain avec le consentement du compositeur. S'inscrivant nettement dans la lignée de l'École de New York cageienne, Mesa revêt un statut ontologique curieux : elle est le produit d'une indétermination passablement radicale. Comme c'est le cas pour l'œuvre de Cage, Mesa et sa reconstruction numérique nous force à repenser l'ontologie et l'épistémologie de l'œuvre musicale.

Mots clés : live electronics; Gordon Mumma ; migration ; musique mixte ; préservation d'œuvres électroniques.
\end{abstract}

\begin{abstract}
This paper reports on a project to produce a reconstruction of a pioneering work of live electronics, Mesa (1966) by the U.S. composer Gordon Mumma (b. 1935). A digital recreation of the work was presented at the Montreal/New Music Festival on 27 February 2017 by the author of this article and his collaborators: Jonathan Goldman (bandoneon), Francis Lecavalier (programming) and Ofer Pelz (electronic performance). This migration entailed a digital recreation of the fundamentally analog processes of the original work, undertaken with the composer's explicit consent. Emerging from Cagean aesthetics, Mesa has a curious ontological status, since it is a product of a fairly radical indetermination. As such, Mesa and its digital reconstruction require an ontological and epistemological reconsideration of the musical work.

Keywords: live electronics; Gordon Mumma; migration; mixed music; preservation of electronic music.
\end{abstract}


En tant que compositeur inscrit dans la tradition expérimentaliste étatsunienne, Gordon Mumma (né en 1935) a très tôt développé une grande habileté dans la production de musique électronique en direct grâce à des appareils qu'il fabriquait et manipulait lui-même. Mumma concevait et interprétait lui-même de cette musique, seul ou bien en collaboration avec des figures centrales de cette tradition comme John Cage, David Tudor ou Robert Ashley. Cet article résume les retombées d'un projet de reconstruction d'une œuvre pionnière de live electronics de Mumma, à savoir Mesa (1966), pour bandonéon (le type de concertina le plus courant dans le tango argentin) et dispositif électronique. Une reconstruction de Mesa a été présentée dans le cadre du festival Montréal/Nouvelles Musiques (MNM) le 27 février 2017 par Jonathan Goldman (bandonéon), Francis Lecavalier (conception informatique et console) et Ofer Pelz (interprétation de la partie électronique). Cette œuvre, fondamentalement analogique, a été reconçue pour un équipement numérique avec le consentement du compositeur. Ce projet relève donc de ce qu'on nomme couramment la " migration ", à l'instar de nombreux projets qui ont vu le jour depuis au moins deux décennies et qui cherchent des solutions aux problèmes de la réinterprétation et de la rediffusion d'œuvres pourvues de dispositifs électroniques d'époques révolues (Wetzel 2006, Boutard, Guastavino et Turner 2013, Bonardi et Barthelemy 2008, Lemouton et Goldszmidt 2016). Pour ne nommer que quelques exemples, on peut évoquer un important projet de reconstruction du Poème électronique (1958) d'Edgard Varèse à l'aide de techniques de réalité virtuelle et de l'audio binaural (Lombardo et al. 2009), ou la reconstitution du code informatique employé par John Chowning dans des œuvres charnières d'informatique musicale comme Stria (1977) ou Turenas (1971-1972) (Zattra 2007, Baudouin 2008, Pottier 2013). Plusieurs de ces projets de migration sont soutenus par un appui institutionnel important, comme c'était le cas pour le projet Intégra, hébergé au Conservatoire de Birmingham jusqu'en 2012, qui a consacré une part importante de ses activités à la migration d'œuvres classiques du répertoire, alors que d'autres se font ad hoc et sans soutien particulier en préparation d'un concert qui souhaite redonner vie à telle ou telle œuvre désormais classique d'électronique en temps réel ${ }^{1}$. Or, la pérennité pose problème même lorsqu'il s'agit d'œuvres beaucoup plus récentes que le Poème électronique, en raison de l'effet pernicieux de l'obsolescence programmée : des systèmes d'exploitation en flux constant font en sorte que des patchs conçus il y a à peine quelques années risquent de ne plus être opérationnels lorsque transposés sur l'équipement d'aujourd'hui - ou même simplement suite à des mises à jour logicielles.

La migration de Mesa présente un niveau supplémentaire de complexité en raison de sa qualité d'ouverture et de son caractère radicalement indéterminé. S'inscrivant dans la lignée de l'École de New York cageienne, Mesa revêt un statut ontologique curieux, car elle est le produit d'une indétermination particulièrement radicale :

1 Les directeurs de recherche de ce projet expliquent que leur objectif est que « the preservation of existing works is made possible by porting the original technology —usually hardware-dependent —onto a software-based environment, and storing all the performance data on a database available online » (www.integra.io, consulté en 2015). 
n'ayant pas de partition, sa durée varie d'exécution en exécution ; il en va de même pour son instrumentation, car si elle a été composée pour bandonéon et dispositif électronique, elle a parfois été jouée par le compositeur et ses complices sur trois harmonicas chromatiques... L'œuvre a aussi connu d'importantes mutations au fil de ses nombreuses représentations depuis sa création en 1966, conséquence de son utilisation en tant que trame sonore de la pièce chorégraphique Place de la Merce Cunningham Dance Company, jouée lors de plusieurs tournées en Europe et en Amérique du Sud. Ainsi, l'équipement de Mesa a évolué au fil du temps, à l'instar du décor de Place, bricolé par les soins de la scénographe Beverly Emmons à partir de détritus (des chaises brisées, par exemple) trouvés autour des théâtres où la compagnie de danse se produisait. Comme dans l'œuvre de Cage, Mesa nous force à repenser l'ontologie et l'épistémologie de l'œuvre musicale en tant que telle, un questionnement que sa migration souligne de façon notable.

Notre hypothèse de départ était que l'œuvre se résume à et s'épuise dans son dispositif - ce qui est peut-être le cas pour toute œuvre indéterminée avec dispositif électronique. Cette position épistémologique est par ailleurs suggérée par Tudor lui-même à propos d'une autre œuvre qui peut être vue comme la " sœur jumelle » de Mesa. Il s'agit d'une œuvre pour bandonéon et électronique que Tudor a composée en 1967, et dont il a affirmé qu'elle n'utilise aucun " composing means since when activated it composes itself out of its own composite instrumental nature ". Affirmer que l'œuvre se résume à son dispositif revient à avancer qu'elle coïncide parfaitement avec les " affordances » des moyens techniques qu'elle utilise. Cette position concorde par ailleurs avec le propos de Thomas DeLio à propos des œuvres les plus multidisciplinaires, collaboratives et indéterminées parmi l'immense catalogue d'œuvres de Cage, la série de ses Variations. À propos de la Variation VI de 1966, écrite " pour une pluralité de systèmes sonores ", DeLio note que : "[T]he structural framework isolated is the very potentiality of raw materials to take on form. Here, a structure is defined solely through the choice of materials and the identification of a range of transformations available as a result of that choice of materials " (DeLio 1993, p. 164).

Si Mesa se résume à son dispositif, une question fondamentale à propos de notre projet de recréation se pose immédiatement : que reste-t-il de l'œuvre d'origine lorsque nous altérons fondamentalement son dispositif en passant de l'analogique au numérique ? Le projet que nous présentons ici se situe à mi-chemin entre d'une part, un projet « authenticiste » qui vise à reconstituer le dispositif de départ tel que le créateur l'a conçu (tout en s'éloignant de cette visée dans la mesure où ce dispositif sera fondamentalement modifié) et, d'autre part, un projet " conservationniste " qui vise à recréer une réalisation sonore (tout en s'écartant du modèle, dans la mesure où ce qui est modélisé n'est pas le résultat sonore d'une version originelle, mais plutôt le fonctionnement du dispositif d'origine). Au cours de la recréation, lorsque ces deux orientations théoriques (authenticiste et conservationiste) entraient en contradiction, nous favorisions la seconde, l'un des buts principaux du projet consistant en 
la présentation de l'œuvre en concert. Or, c'est bien le souci de la migration, et non celui de la fidélité historique, qui allait assurer la livraison d'un concert réussi, et ce un demi-siècle après la création de cette œuvre technologique. Ce compromis entre une volonté de recréer les circuits d'origine employés par Mumma et celle de proposer un nouveau dispositif qui s'inspire soit de ces circuits soit du rendu sonore est en phase avec la démarche créatrice empruntée par le compositeur lui-même à l'époque de Mesa, comme en témoigne une conférence de 1967 dans laquelle il affirme que :

Sometimes I am looking for a certain kind of sound modification, and I work on various circuits until I have achieved that result. Other times in casually experimenting with different configurations of circuits I may chance upon some novel sound effect that becomes the germinating idea for a piece of music (Mumma 2015, p. 44).

Après une présentation de Mesa et une mise en contexte du projet de migration, cet article présentera un exposé des éléments saillants de l'atelier de musique contemporaine de Mumma. Nous proposerons ensuite une présentation de l'approche adoptée pour la migration de l'œuvre, ainsi qu'un questionnement autour des différentes approches de l'interprétation de l'instrument numérique adoptées en contexte de concert. En effet, avant de pouvoir procéder à la traduction numérique de l'œuvre, il fallait d'abord comprendre dans quelle mesure et dans quel sens Mesa pouvait être considérée comme une œuvre au sens fort, et quelles étaient ses caractéristiques essentielles ou contingentes. Un questionnement épistémologique a dès lors accompagné notre démarche, et le produit final - notre interprétation de l'œuvre en concert - a été, à son tour, producteur d'une autre série de questions sur le statut ontologique d'une œuvre aussi diaphane que Mesa.

\section{CONTEXTE}

Mesa est historiquement marquante non seulement pour ses innovations techniques, mais aussi en raison de l'importance capitale qu'elle a eue dans le cheminement artistique de son premier interprète, David Tudor. La création de Mesa se trouvait à un point de transition entre sa vocation de pianiste mythique de l'avant-garde et sa carrière de compositeur de musique électronique, aboutissant notamment aux dispositifs électroniques très personnels qui ont fourni la trame sonore de la chorégraphie Rainforest de la Compagnie Cunningham en 1968³. Entre ces deux étapes, Tudor s'est consacré à l'apprentissage du bandonéon, dont il exécute la partie dans Mesa. L'interprète mythique du répertoire contemporain de Karlheinz Stockhausen, Pierre Boulez, Christian Wolff, Earle Brown, Sylvano Bussotti et surtout de John Cage, a découvert l'instrument grâce à son amitié avec le compositeur Mauricio Kagel (1931-2008), natif de l'Argentine et installé à Cologne en 1957, là même où Tudor s'est souvent rendu afin de collaborer avec Stockhausen en studio ou en concert. Kagel et Tudor commencent à travailler ensemble vers 1959 et le compositeur argentin lui compose 
une pièce solo pour bandonéon, Pandorasbox (Bandoneonpiece) en 1960, dont Tudor exécutera la première audition étasunienne en 1965.

Par la suite, Tudor commence à commander d'autres pièces pour son nouvel instrument aux compositeurs faisant partie de son cercle d'expérimentalistes américains, dont Mumma, le plus adepte de son entourage de la fabrication de circuits électroniques pour le traitement du son en temps réel. Parmi ces pièces, on compte le Duo for Accordion and Bandoneon (1964) de Pauline Oliveros, ou la Piece for Bandoneon and Strings (1964) de Stanley Lunetta, dans laquelle les cordes ne sont pas celles des violons ou des altos, mais des fils électriques au bout desquels se trouvaient des microphones posés à même le corps de Tudor. Pour Mesa, qui a été créée à la Fondation Maeght à Saint-Paul-de-Vence (France) en août 1966, le compositeur a exploité un dispositif électronique de traitement du son en temps réel, avec une diffusion quadriphonique. Alors que Tudor jouait la partie de bandonéon, Mumma contrôlait le dispositif électronique au moyen d'une console située sur la scène.

Après l'aventure de Mesa, Tudor a lui-même composé une œuvre de grandes dimensions pour bandonéon et électronique, Bandoneon! (a combine), que nous avons qualifiée plus haut de "sœur jumelle » de Mesa. L'œuvre est créée en octobre 1966, dans le cadre du projet 9 Evenings. Theatre and Engineering à 1'Armory de New York, un événement qui proposait des collaborations entre des artistes tels John Cage et Robert Rauschenberg et les ingénieurs des Bell Laboratories, dans le but d'encourager les artistes à se servir dans leur pratique artistique des nouvelles technologies audiovisuelles inventées au laboratoire. Ce spectacle multimédia se servait du bandonéon pour provoquer un grand nombre d'événements sonores et visuels, notamment des motifs graphiques.

\section{Le « Cooperative Studio for Electronic Music » de Gordon Mumma}

À 1'hiver 1952, Gordon Mumma s'inscrit à l'école de musique de l'Université du Michigan, situé à Ann Arbor. Il y étudie brièvement avec Ross Lee Finney et Leslie Bassett avant d'abandonner le programme de baccalauréat (licence de premier cycle) à l'automne 1954. Il reste néanmoins dans cette ville universitaire et poursuit à titre indépendant de nombreux projets de création, souvent associés aux activités de création de l'université, le plus significatif étant une collaboration à partir de 1957 avec le compositeur Robert Ashley au sein du Space Theatre, un espace de projection et de performance conçu pour les jeux de lumière par le peintre et professeur de beaux-arts à l'Université du Michigan, Milton Cohen. Peu après avoir assisté à une conférence donnée par Stockhausen, invité à l'école de musique en 1958, au cours de laquelle le chef de file de l'avant-garde européenne évoque la figure du compositeur indépendant, Mumma fonde un studio de musique électronique avec le compositeur Robert Ashley, qui devient le "Cooperative Studio for Electronic Music ». La première œuvre de Mumma pour sons électroniques sur bande, Vectors, date de 19594. En 1960, Mumma

4 Dans ce paragraphe, nous suivons largement le bilan biographique proposé par Michelle Fillion dans son " préambule » aux écrits de Mumma (Fillion 2015). 
fonde avec l'ingénieur William Ribbens la compagnie Cybersonics pour la production d'appareils électroniques destinés à la musique. C'est aussi à Ann Arbor que Mumma cofonde en 1961 le festival ONCE, haut lieu de musique expérimentale. C'est lors de ce festival que Mumma croise souvent Tudor ; les deux musiciens se lient d'amitié. C'est pendant l'édition de 1965 que Tudor lui commande une ouvre pour bandonéon et électronique qui allait devenir Mesa. Au cours des années 1960, Mumma commence à composer des œuvres conçues dans le Cooperative Studio et avec des appareils conçus par sa compagnie, qu'il qualifie de " cybersoniques ». Il s'agit d'une approche qu'il définit comme un processus électronique où le son est modifié " par les caractéristiques directement issues du son lui-même » (Fillion 2015, p. xxix), un principe au cœur des traitements de Mesa. Parlant de Mesa dans sa conférence de 1967, Mumma note que : "All of the control signals for these sound modification functions are derived from the sounds of the bandoneon. [...] Because the control signals are automatically derived from the sound materials themselves, I call the process and the music 'cybersonic'" (Mumma 2015, p. 48).

De façon générale, Mumma ne sépare jamais la lutherie électronique du processus de création dans ses œuvres mixtes. Comme il l'écrit :

My electronic music equipment is designed to be part of my process of composing music. I am like any composer who builds his own instruments, though most of my 'instruments' are inseparable from the compositions themselves. My 'end-product' is more than a package of electronic hardware: it is a musical performance for a live audience (ibid., p. 43).

Mumma situe son activité créatrice sur le plan de ce qu'il nomme des « concepts-systèmes ", qui englobent le dispositif ( "configurations », dans les termes de Mumma) en tant que totalité :

I am concerned primarily with 'system-concepts' - configurations that include sound sources, electronic modification circuitry, control or logic circuitry, playback apparatus (power amplifiers, loudspeakers, and the auditorium), and even social conditions beyond the confines of technology. I suggest that the most important creative aspect of live-performance electronic music technology is not this or that circuit innovation but rather the total configuration itself (ibid., p. 44).

Depuis ses débuts jusqu'à la fin des années 1950, ce savoir-faire et cette activité ont évolué, du moins selon Mumma lui-même, qui a jeté un regard rétrospectif en 1967 sur sa production en matière de musique mixte en prenant en exemple quatre œuvres de son catalogue en ordre croissant de sophistication, à savoir Medium Size Mograph (1963) pour piano à deux exécutants, Hornpipes (s.d.), une œuvre pour cor ultérieurement retirée, Diastasis, as in Beer (1967) pour deux guitares électriques, et finalement Mesa (voir ibid., p. 43-49). Alors que dans Medium Size Mograph le circuit a essentiellement servi à atténuer le transitoire d'attaque du piano et à dilater la durée de sa désinence, celui de Hornpipes a relié un micro placé dans le cor et d'autres micros situés dans plusieurs tuyaux de plomberie en métal à un haut-parleur, créant un dispositif de retour du signal électronique (" electronic feedback system »). Dans Diastasis, as in Beer, Mumma a construit une relation d'interdépendance entre les deux guitaristes en faisant en sorte que l'amplitude du signal de l'un détermine la fréquence du signal de l'autre, et ce grâce à un système de détection de l'amplitude de l'enveloppe qui 
est sensible à la fréquence. Enfin, le dispositif de Mesa a dépassé les trois précédents en complexité, d'abord de par son déploiement en quadriphonie, mais aussi par son utilisation de traitements comme le "spectrum transfer" (translation de fréquence avec égalisation), le "multiplier» (translation du spectre par des nombres entiers plus égalisation), le "product valve " (modulation d'amplitude et de phase), le "formant modulation " (filtrage en peigne contrôlé par potentiomètre), etc.

Le savoir-faire de Mumma est tel qu'il publie un article en 1964 dans la principale publication américaine consacrée à l'ingénierie du son, le Journal of the Audio Engineering Society (Mumma 1964). Une relecture éclaire l'approche de la musique électronique préconisée par le compositeur. L'article définit la musique électronique comme « une musique composée directement sur bande magnétique par des moyens électroniques $^{5}$ » (Mumma 2015, p. 14), et produite la plupart du temps, précise Mumma, dans des laboratoires universitaires ou des radios d'État. Mais le compositeur ayant désormais la possibilité de construire son propre studio, il en propose les principes de base en tenant compte de contraintes budgétaires propres à un individu compositeur ne jouissant pas d'un soutien institutionnel. Il répartit les fonctions du studio en quatre grandes catégories :

1) General manipulation apparatus: tape recorders, amplifiers, mixers, etc.

2) Sound sources: oscillators, etc.

3) Modification apparatus-filters and equalizers, transposition devices, gating and envelope control and reverberation

4) Accessories: power supplies, monitoring and analysis equipment such as meters, loudspeakers, and the oscillosocope; splicers, the bulk eraser, etc. (ibid., p. 15).

C'est par rapport à l'élément 3) que son approche est particulièrement intéressante. Dans une sous-section titrée "Modulation ", Mumma parle des "basic processes of amplitude modulation and frequency (or phase) modulation" (ibid., p. 18), énumérant les manières dont ces processus peuvent servir un compositeur de musique électronique : "gating (orkeying) and envelope control; volume compression and expansion; frequency spectrum transposition, inversion, compression, and expansion; and complex spectrum production " (ibid.). Mumma précise que les appareils adaptés pour ces fins ne sont pas disponibles sur le marché, mais ne sont "ni difficiles ni coûteuses à fabriquer ${ }^{6}$ ". À cette fin, il évoque d'une part des "passive semiconductor modulators » et des " active modulators ", ces derniers étant parfois pris en charge par des "transistor emitter modulators" (le type de composant dont Mumma se servira dans Mesa). Il conclut ainsi : "The various types of passive and active balanced modulators, in conjunction with frequency filtering and wideband phase-shifting accessories, constitute an extremely important area of sound modification apparatus for electronic music composition » (ibid., p. 15).

L'article de Mumma, rédigé à l'intention des membres de la Audio Engineering Society aide à comprendre les composants principaux du dispositif de Mesa, dont le schéma électronique se trouve à la figure 1 .

5 « $[A]$ music that is composed directly on magnetic tape by electronic means ».

6 "Neither difficult nor expensive to build». 


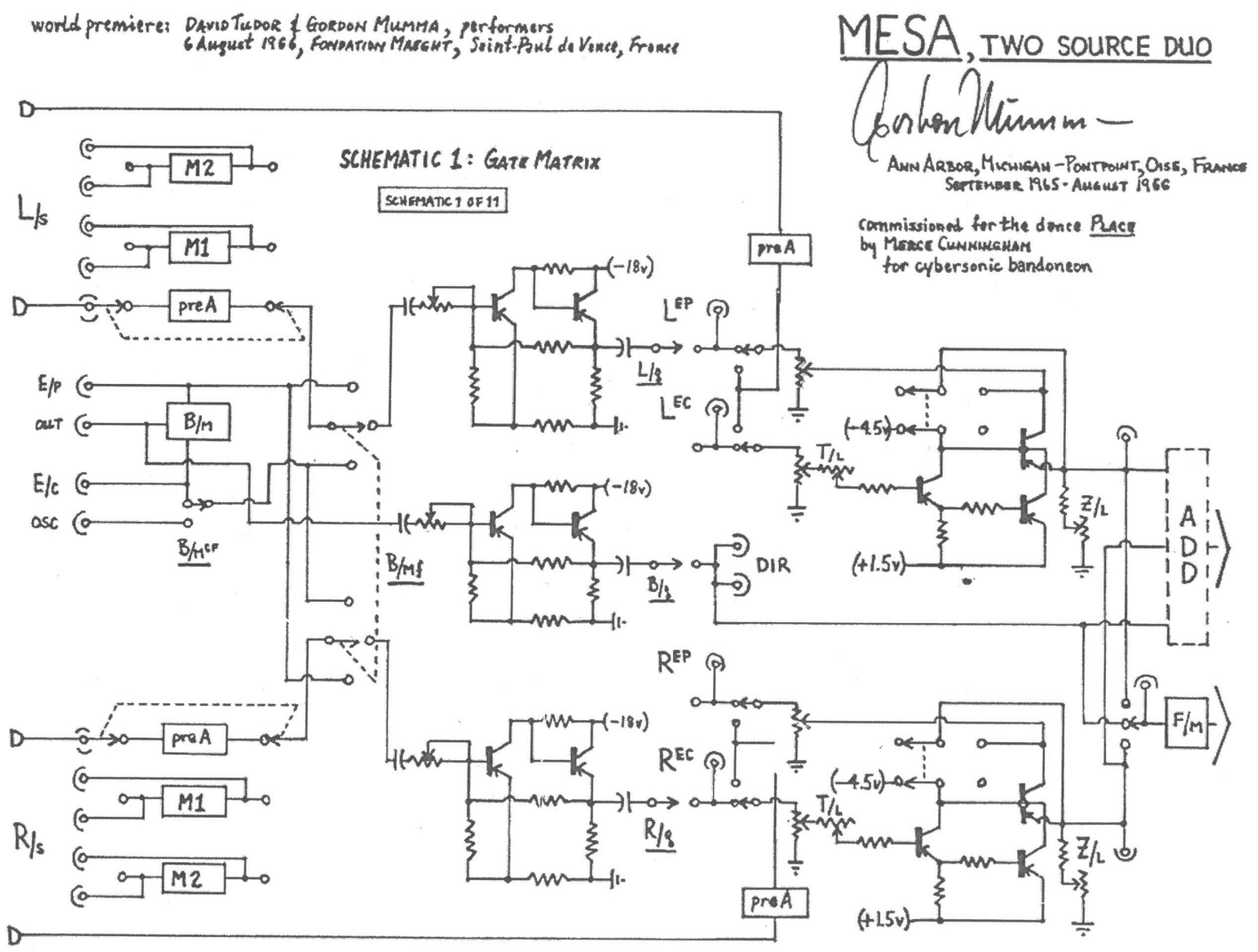

Figure 1 : Schéma du dispositif électronique de Mesa ; reproduit avec l'aimable autorisation de Gordon Mumma.

Un regard rapide sur le schéma confirme la présence d'un oscillateur (bord gauche, milieu) et de transistors bipolaires («Bipolar junction transistor» ou BJT) dont le symbole se trouve à la figure 2 .

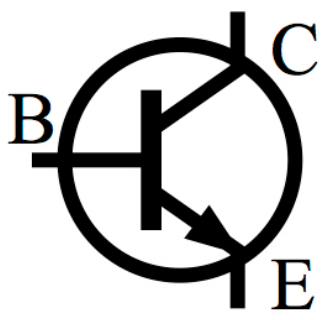

Figure 2: Symbole schématique d'un transistor bipolaire.

La présence de transistors est significative, car le titre de l'œuvre renvoie entre autres au transistor Mesa développé par Bell Labs en 1955 et mis sur le marché en 1958. Comme l'explique Mumma dans une notice de l'œuvre, le titre évoque les " aspects of its binary musical architecture, the structural geometry of monolithic semiconductors used in its electronic circuits, the contours of its cybersonic thresholds, and the expansive, eroded, mesa landscapes of the south-western United States" (Mumma 2002, livret). Or, une des fonctions des transistors se trouve dans la modulation en anneau, qui de 
façon générale est effectuée de façon analogique soit à l'aide de diodes, soit à l'aide de transistors (voir Hoffmann-Burchardi 2009). En effet, la modulation en anneau constitue le traitement principal à l'œuvre dans Mesa. Rappelons que cet effet consiste essentiellement à multiplier un signal porteur (carrier) par un autre signal modulant (modulator). Les partiels du signal porteur original sont ainsi remplacés par deux autres partiels égaux à la somme et la différence de la fréquence modulante. Lorsque le signal est plus complexe, cette opération est effectuée sur chaque partiel du signal porteur pour chaque partiel du signal modulant. C'est par la modulation en anneau que l'œuvre " se compose par elle-même, à partir de sa propre nature instrumentale mixte ", selon les termes de Tudor évoqués plus haut.

L'autre fonction des transistors BJT dans le schéma est celle de gating, où, comme l'a décrit un critique commentant la performance de Mesa lors de du premier Festival of Live Electonic Music (FLEM) en 1967 : «à partir de certains seuils, le son se transforme en un dispositif de transformation » (Johnson 2011, p. 117). C'est grâce au dispositif de gating que seuls des sons émis de la source (le bandonéon) ayant des caractéristiques particulières (fréquences, intensités, timbre, etc.) déclenchent des traitements électroniques. Or, le fait que le titre de Mesa renvoie à un transistor, qui constitue la partie essentielle de son dispositif électronique, tend à confirmer l'hypothèse que l'œuvre se confond avec son dispositif.

Comme nous l'avons déjà mentionné, Mesa est conçue et composée pour une diffusion quadriphonique. Ainsi, outre la présence de transistors, le schéma montre clairement la présence de quatre microphones (deux du côté droit de l'instrument étiqueté $[\mathrm{R} / \mathrm{s}]$ et deux du côté gauche $[\mathrm{L} / \mathrm{s}]$ ) auxquels correspondent deux sorties reliées à quatre haut-parleurs. Le son produit par le modulateur en anneau est dupliqué et envoyé sur quatre canaux, chacun assigné à un haut-parleur dans la salle. L'amplitude, ou l'intensité de chacun de ces canaux de sortie est modulée par l'amplitude du signal provenant de l'un des microphones collés sur le bandonéon, grâce à des suiveurs d'enveloppes ${ }^{7}$. Ce système permet donc de créer une spatialisation augmentée, puisque les mouvements d'amplitude sonore sont fidèlement reproduits sur chaque haut-parleur. En entretien, Mumma insistait sur l'importance de ce dispositif à quatre canaux :

And there was a thing I did which was really good, because, you know, it's already stereo, but I then 'stereo' each side, that is it's quadraphonic: top and bottom on each side. And I used special microphones so that you get different sounds on each side, the resonance things are all different. And when we performed this with the Cunningham Dance Company every chance we had, it was a quadraphonic arrangement, so that you had this strange thing that usually came out of one source, but it was all over the place. All live. And expanding that spectral and spatial aspect was very important, because then it made it possible for Tudor or me when I played it, to not have to work hard with sound, at making all kinds of things; I mean little changes here or there could make whole big things. That was a big part of the concept (Goldman 2008).

7 Par exemple, la sortie 1 voit son amplitude modulée par l'amplitude du signal du micro 1, le canal 2 par le micro 2, etc. 
Dans une conférence rédigée l'année après la création de Mesa, Mumma situait le besoin de spatialisation quadriphonique dans une volonté de transparence et d'intelligibilité vis-à-vis de l'auditeur :

In order to achieve the frequency spectrum and sound density of Mesa, as well as to control aspects of the spatial perception of the audience and still maintain clarity in the musical continuity, I deploy related portions of the sound through different loudspeakers in the auditorium (Mumma 2015, p. 46).

Mumma a également publié un autre schéma du dispositif de Mesa, qui, quoique moins détaillé, montre clairement la façon dont le signal de chaque micro d'un côté de l'instrument vient s'ajouter à celui d'un micro de l'autre côté, créant une situation où un côté de l'instrument module le son de l'autre (figure 3).

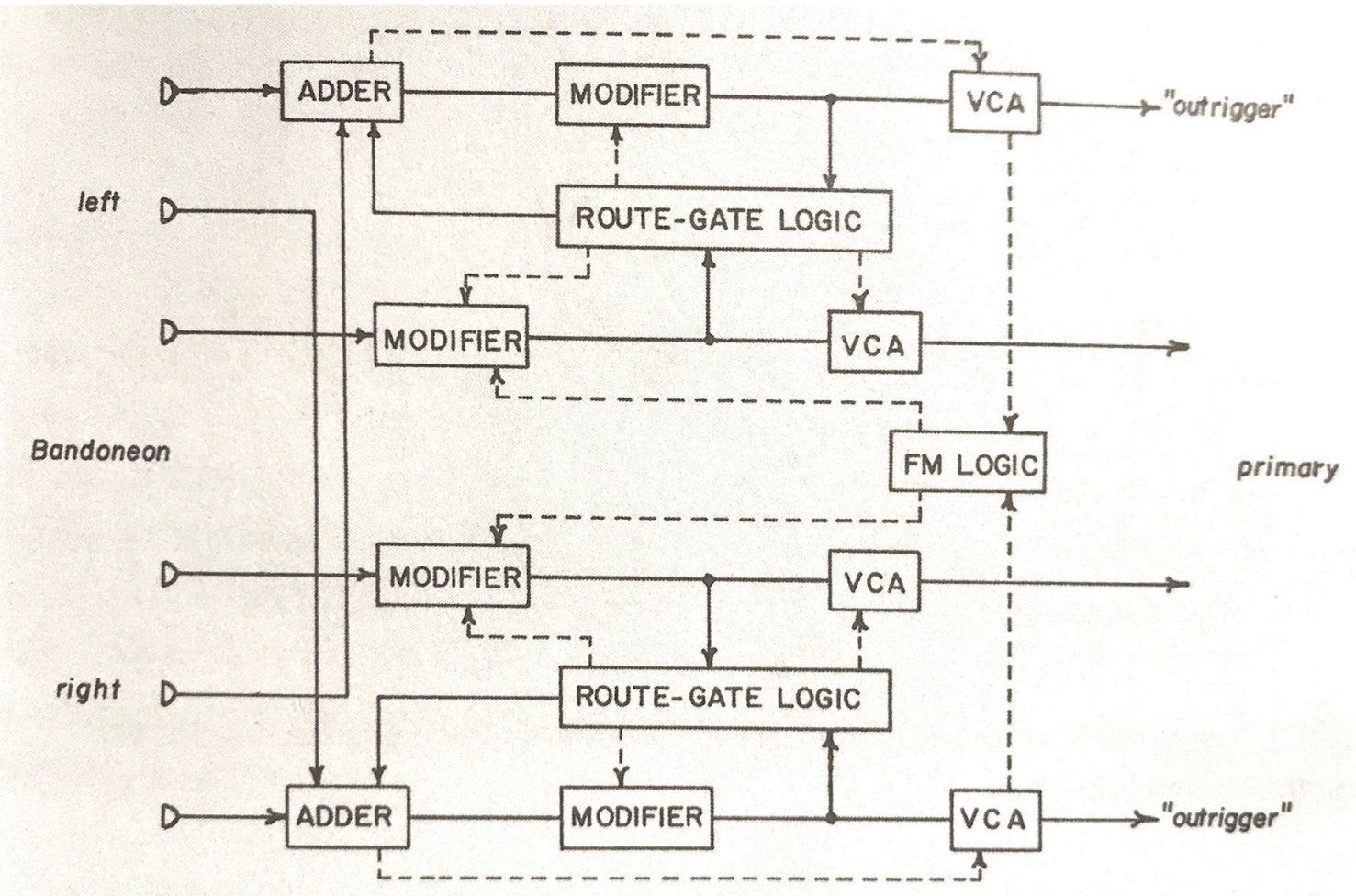

Figure 3 : Dispositif général pour Mesa, tiré de Mumma 2015, p. 47 ; reproduit avec l'aimable autorisation de Gordon Mumma.

La figure 3 montre la présence d'atténuateurs de tension contrôlés (« voltage-controlled attenuators», VCA) dans le circuit de Mesa, un aspect sur lequel Mumma insiste dans sa conférence de 1967. Citant la difficulté d'étendre l'ambitus dynamique du bandonéon, il précise qu'il a inclus dans son circuit des moyens de contrôler les enveloppes. Pour ce faire, il fait appel à quatre "voltage-controlled attenuators (VCA) developed in collaboration with William Ribbens in Ann Arbor» (ibid., p. 46). De façon générale, le schéma montre aussi le savoir-faire d'un compositeur de musique électronique de tendance expérimentaliste à cette époque, impliquant une compréhension des circuits et même la capacité d'en construire soi-même, souvent à partir de pièces trouvées dans des 
magasins de surplus d'armée ; cette pratique DIY ( do it yourself») fait ainsi partie intégrale des options esthétiques empruntées dans l'œuvre, et dont la migration se doit de tenir compte ${ }^{8}$.

\section{TRADUCTION NUMÉRIQUE D’UNE EUVRE ANALOGIQUE MIXTE}

L'idée de redonner vie à Mesa remonte à 2007 lorsque Jonathan Goldman intègre le Département de musique de l'Université de Victoria, en Colombie-Britannique, où habite Mumma. Apprenant que Goldman joue du bandonéon, Mumma lui raconte ses expériences avec cet instrument et tout particulièrement la genèse de Mesa en collaboration avec Tudor. Goldman a par la suite réalisé un projet de recherche sur Tudor et le bandonéon (avec la précieuse collaboration de Mumma) qui a abouti à un article scientifique paru dans la revue American Music (Goldman 2012). Ayant intégré la Faculté de musique de 1'Université de Montréal en 2013, Goldman souhaite étendre la recherche de ce projet au domaine de la recherche-création en présentant une reconstruction de Mesa avec l'aide du secteur de musique numérique de sa nouvelle institution. C'est ainsi qu'un collègue l'a mis en contact avec Francis Lecavalier, alors étudiant au programme de baccalauréat en musique numérique, qui est aussitôt devenu l'auxiliaire de recherche de Goldman en vue de ce projet. Profitant d'un appel à projets du festival Montréal/Nouvelles Musiques (MNM), Goldman a proposé un concert d'une heure qui marquerait les 50 ans du First Festival of Live Electronic Music (Université de Californie à Davis, 1967), un festival qui mit à l'honneur David Tudor et qui vit l'une des premières exécutions de Mesa. Goldman a invité la pianiste réputée Brigitte Poulin à se joindre au projet pour interpréter des œuvres pour piano de Mumma ainsi que des extraits des Sonatas and Interludes pour piano préparé de Cage, dans le but de signaler les liens reliant Mumma à l'École de New York. Le compositeur Ofer Pelz, alors doctorant en composition à la Faculté de musique de l'Université de Montréal, a été recruté non seulement pour contrôler le dispositif électronique de Mesa en concert, mais aussi pour composer des transitions acousmatiques qui allaient être jouées entre les pièces. Lecavalier a ensuite élaboré le nouveau dispositif de Mesa en collaboration avec Mumma, avec lequel il correspondait par courriel. Initialement, Mumma devait participer au concert en ajoutant le son d'un harmonica chromatique à celui du bandonéon de Goldman, mais des soucis de santé l'ont empêché d'y assister. Un enregistrement du concert lui a été néanmoins envoyé, et Mumma a réagi positivement à cette nouvelle incarnation de son œuvre.

Outre les schémas électroniques de Mesa (figures 1 et 3), la reconstruction de l'œuvre a été facilitée par l'accès à plusieurs autres sources de documentation dont :

1) Deux enregistrements réalisés par Mumma et Tudor : 1'un tiré d'un disque réalisé dans le studio de CBS sur la 30 avenue de Manhattan en 1967 et paru l'année suivante, et une captation en direct d'un spectacle de Place, enregistrée à Paris

8 Cette approche DIY chez Mumma ainsi que chez Cage et Tudor a été explorée récemment dans Hartman 2019. 
le 9 novembre 1966 et paru sur un coffret de disques compacts en 2010. Il est significatif que les durées des deux enregistrements diffèrent significativement, l'un étant de $22 \mathrm{~min} 50 \mathrm{~s}$, et l'autre de $19 \mathrm{~min} 38 \mathrm{~s}$ (extraits audio 1 et 2).

Extrait audio 1 et 2 : Deux enregistrements de Mesa par Gordon Mumma et David Tudor : 1) enregistrement de spectacle, 19 min 38 s, extrait du coffret Music for Merce (1952-2009), New World Records 807122, 2010, enregistré à Paris en 1966 ; écouter l'enregistrement complet ; 2) enregistrement studio, 22 min $50 \mathrm{~s}$, extrait du LP A Second Wind For Organ, Odyssey 32160158,1968 ; écouter l'extrait.

2) Un « plan » temporel de l'enregistrement studio, préparé par Mumma post facto, sorte de hörpartitur assez peu détaillée (figure 4). En préparant ce schéma, Mumma a été frappé par l'apparente forme bipartite de l'œuvre, avec une segmentation significative survenant au centre exact de l'œuvre. Toutefois, la nature des événements constituant les repères de la charpente formelle n’y est pas spécifiée.

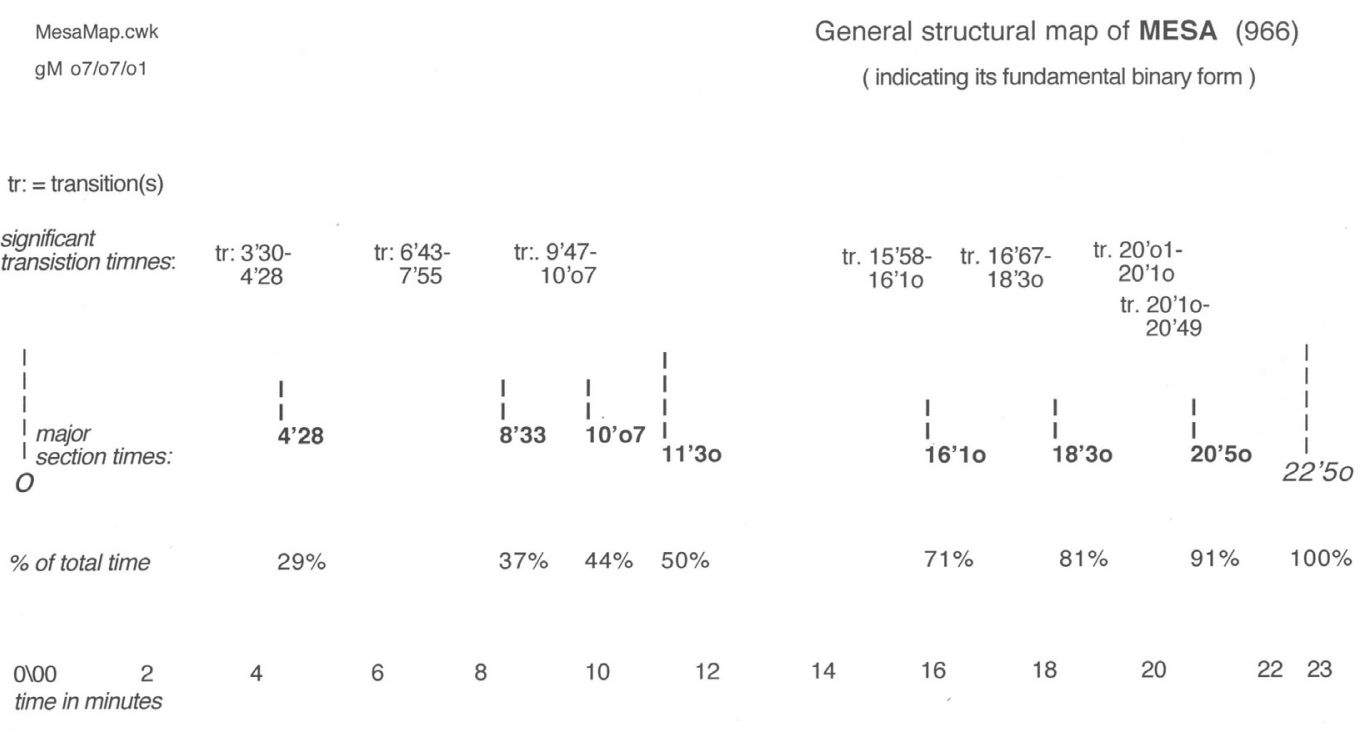

\footnotetext{
Timings from 1967 August recording by David Tudor (bandoneon) and Gordon Mumma (cybersonics) at the CBS 30th St. Studio, New York City. ( recordings issued on CBS Odyssey LP 2316-0158, CBS France LP S-346 1065, Lovely Music LP 1092, Tzaidk CD 7074 )

Composition commissioned for Merce Cunningham's choreography PLACE, premiere performance on 1966 Aug. 6, Fondation Maeght, St. Paul de Vence, France.
}

Figure 4 : Plan temporel de l'enregistrement studio de Mesa, préparé par Gordon Mumma à des fins pédagogiques ; reproduit avec l'aimable autorisation de Gordon Mumma.

3) Un film sonore d'une performance de Place (dont un extrait peut être visionné ici) ayant eu lieu à Buffalo, New York, datant de 1968. Si ce film est d'une valeur documentaire précieuse, il renseigne moins sur le son de Mesa, car la partie sonore a été endommagée - et le son y figure avec d'importantes distorsions, battements $\mathrm{ou}$ « wobble».

3) Des échanges de courriel avec Mumma tout au long du processus. Goldman a approché le compositeur en novembre 2015 pour savoir s'il était favorable à l'idée de représenter Mesa. Recevant une rapide réponse favorable, la correspondance se poursuivit jusqu'à la date du concert et au-delà. Dès le $1^{\text {er }}$ juin 2016, Goldman 
envoyait à Mumma un premier schéma (un prédécesseur à la figure 5) préparé par Lecavalier, en lui demandant de faire part de ses retours. En général, les réponses de Mumma ne concernaient pas directement le schéma, mais donnaient plutôt lieu à d'autres précisions remémorées grâce au support du schéma (comme la présence de tel ou tel micro qui ne figure pas sur le schéma officiel de l'œuvre). Voirl'annexe pour une chronologie et un résumé des échanges avec le compositeur).

Si l'œuvre se résume essentiellement à son dispositif, comprendre ce en quoi consiste son appareillage électronique constitue un travail d'exégèse fondamental pour la représentation de Mesa. Or, les descriptions qui nous ont été fournies par le compositeur se sont avérées des plus utiles à ce travail de recréation. Nos quelques échanges avec Mumma se sont faits par courriel, mais même ce moyen par trop imparfait nous a permis de clarifier considérablement la nature du dispositif. Comme le compositeur s'est vite révélé favorable à l'idée du projet, nous avons pu obtenir sans difficulté des précisions de sa part. Nous transmettions nos brouillons de schémas conceptuels et nos explications au fur et à mesure, et il nous répondait avec ses commentaires, ses corrections, ses explications et ses précisions.

Dans un courriel capital reçu le 4 avril 2016, Mumma précise que ce qui est représenté dans le schéma électronique qui se trouve à la figure 5 " are only functional system blocks" lesquels " are likely to be useless for rebuilding the equipment with analog circuit components ». Ce qui compte, selon le compositeur, ce sont les modules insérés dans le dispositif général figurant dans le schéma et qui incluent les cinq éléments suivants :

[1] Ring-modulators,

[2] Band-pass filters,

[3] Envelope-followers with voltage control of gains, etc.

Miscellaneous external equipment:

[4] Mixers > sound-distributors ( power amplifiers),

[5] Sine/square/triangle oscillators (for testing and balancing the sound levels without out the bandoneon (inputs, etc.) (courriel du 4 avril 2016).

C'est aussi dans ce courriel que Mumma explique quels sons servaient de sons porteurs et de sons modulants dans la modulation en anneau :

The 'carrier' frequency input was also from the bandoneon (or harmonica) in performances, but only from a single key-pressed sound on one or the other side of the bandoneon. If multiple keys were used on both sides of the instrument, a narrow-band filter could be applied to the signal before the carrier input to the ring-modulator. In a few instances a broad-band carrier signal was used to further 'thicken' the resulting sound-output (ibid.).

Toujours grâce à ce même message, nous avons appris l'emplacement des microphones, à savoir la présence de deux microphones contact piézoélectrique de chaque côté du bandonéon, en plus d'un microphone cravate ("small 'air-mic' or lavalier-type ») de chaque côté. Ces deux derniers microphones étaient employés pour capter et diffuser le son non traité du bandonéon qui se mélangeait ensuite au son traité («they were used only as 'fade-through' sources for mixing of the unprocessed sounds during live-performances »; ibid.). Ces deux micros fournissaient ainsi une amplification légère du son du 
bandonéon, une sonorité curieusement absente de l'enregistrement studio réalisé par Mumma et Tudor, mais pas de l'enregistrement parisien capté en direct.

Ainsi, il apparaît que le dispositif de Mesa a essentiellement permis de faire moduler le son de n'importe quel micro piézoélectrique par le son de n'importe quel autre. Un filtre passe-bande étroit était également présent à l'entrée du modulateur en anneau, mais il n'était pas nécessairement allumé : le soin du contrôle de celui-ci était laissé à l'interprète du dispositif, pour réduire le spectre du son entrant lorsque ce dernier s'avérait trop dense.

Suite aux échanges avec Mumma et à l'étude des documents énumérés plus haut, Lecavalier, en tant que concepteur informatique du projet, a formulé un schéma pour la reconstruction numérique de Mesa à travers plusieurs brouillons dont le deuxième se trouve à la figure 5 .

$19 / 07 / 2016$
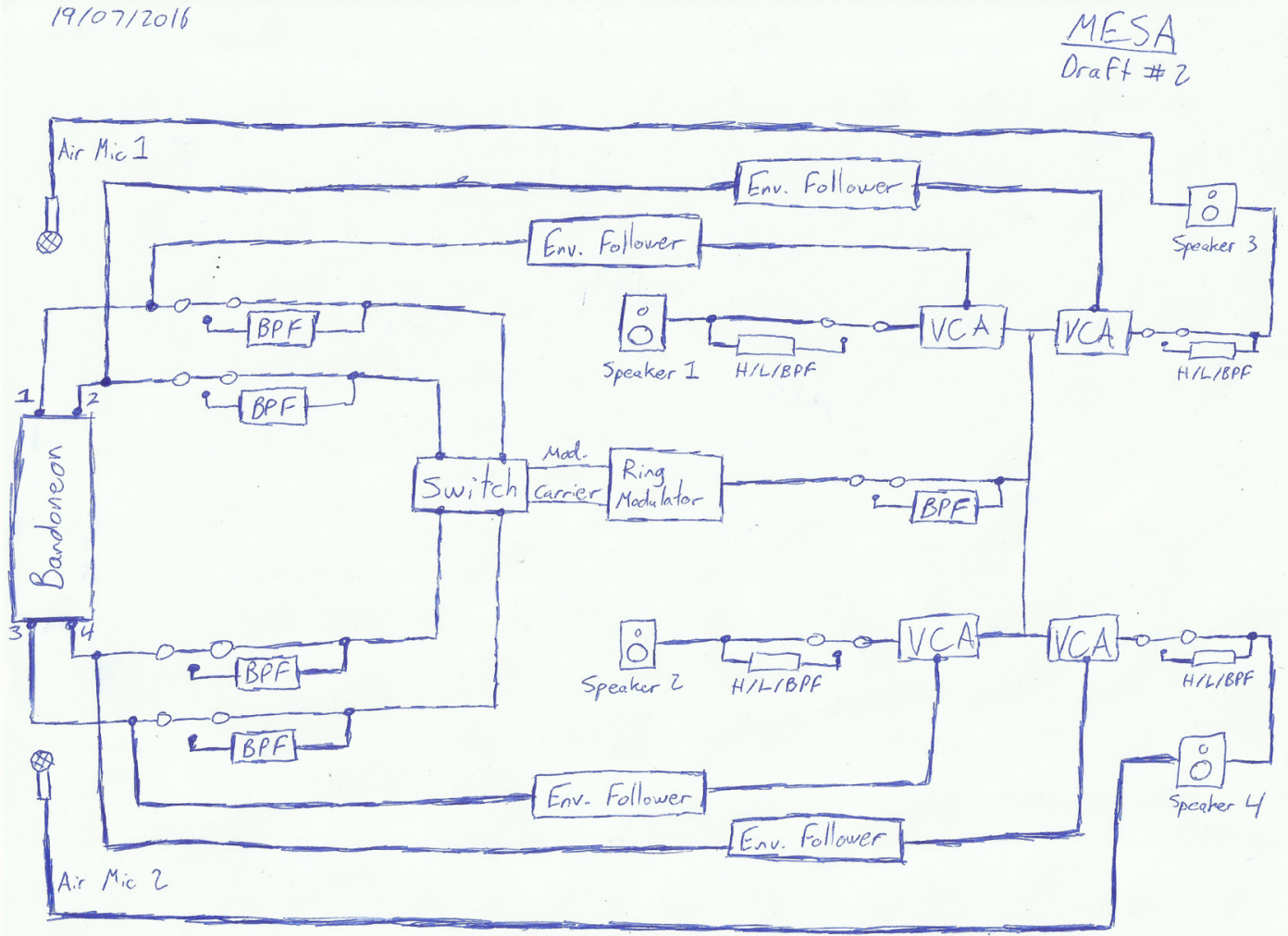

Figure 5 : Schéma préparé par Francis Lecavalier en prévision du concert, $2^{\gtrless}$ version.

Chaque brouillon a été envoyé au compositeur, qui en validait ou en critiquait des éléments. Lecavalier s'efforçait d'adopter une approche " historiciste " à cette reconstruction, le but étant de recréer le dispositif et les sonorités d'origine dans un objectif de fidélité et de respect. Étant donné que le dispositif constituait le cœur même de l'œuvre, une telle approche s'imposait : trop de modifications lui auraient fait perdre son identité. Par exemple, Lecavalier a résisté à la tentation d'ajouter de trop nombreux traitements numériques au dispositif, comme de la granulation, du ré-échantillonnage et autres techniques numériques courantes de nos jours, les jugeant superflus et risquant de faire dévier de l'essence de l'œuvre, aussi ineffable 
que celle-ci puisse paraître. L'élégante simplicité du traitement lui semblait essentielle à son caractère.

Lecavalier a donc recréé le plus exactement possible le dispositif figurant à la figure 5 à l'aide du langage Python (version 2.7). Une application $M E S A$ a été développé selon une approche orientée-objet. L'application est divisée en plusieurs classes, chacune représentant une fonctionnalité précise de l'application. Ceci facilite la réutilisation du code, et permet de créer une architecture modulaire facile d'entretien. Le code complet de l'application est disponible en ligne sur un dépôt GitLab dédié. Le lecteur peut y accéder et explorer le code par lui-même. Ce dernier est bien documenté et facile d'accès pour quiconque connaît le langage Python et les bases de la programmation orientée-objet. Le traitement de signal audio est effectué à l'aide de la librairie Pyo, développée à la Faculté de musique de l'Université de Montréal par Olivier Bélanger. Cette librairie, écrite en langage $\mathrm{C}$ et à la base de logiciels de traitements sonores comme Cecilia et Soundgrain, permet de faciliter la création de chaînes de traitement de signal. Chaque classe de l'application tire donc parti d'un ou plusieurs objets mis à disposition par la librairie Pyo. Par exemple, la classe InputLine est une abstraction représentant une entrée audio du modulateur en anneau. À l'intérieur de cette classe, se trouvent les objets Pyo nécessaires pour effectuer le traitement de signal qui doit avoir lieu avant la modulation en anneau.

Voici à quoi ressemble la chaîne de traitement de signal contenue dans InputLine. Le signal envoyé dans l'entrée audio de la carte de son est récupéré par l'objet Pyo Input. Le signal sortant de Input est envoyé dans un objet InputFader, permettant d'effectuer des fondus enchaînés avec d'autres signaux audio de manière propre et sans artefacts. L'objet $B u t B P$ récupère le signal de InputFader et lui applique un filtre passe-bande, paramétrable par l'interprète. Le signal se dirige ensuite dans FreqShift, qui permet d'effectuer la translation de fréquence. Le signal se divise ensuite en deux : il se dirige dans PeakAmp, qui permet de mesurer les pointes d'amplitude sonore pour alimenter les vumètres de l'application, ainsi que dans Follower2, un suiveur d'enveloppe dont la sortie est utilisée pour moduler le signal de sortie du modulateur en anneau. Autre exemple : la classe MidiServer tire parti de l'objet MidiListener de Pyo pour définir des fonctions callbacks qui seront appelées lorsque des messages MIDI sont détectés par l'application.

Le cour de l'application se situe au sein de la classe RingMod: cette classe définit la fameuse modulation en anneau si fondamentale dans Mesa. Elle utilise des objets Pyo Sig, qui représentent les signaux audio porteurs et modulants obtenus à partir d'objets InputLine (définis plus hauts). Une simple multiplication des signaux y est effectuée afin d'obtenir l'effet désiré. Le signal résultant du modulateur est dirigé dans quatre objets de la classe OutputLine. Cette classe définit le traitement de signal apporté à la sortie du modulateur en anneau. Il incorpore les objets Pyo Disto (distortion), InputFader (qui est modulé par la sortie du Follower2 d'un objet InputLine associé), Compress (compression), Clip (limiteur de signal) et PeakAmp. L'application MESA est donc constituée d'un assemblage d'objets Pyo, regroupés et ordonnés à l'intérieur de classes définies dans plusieurs fichiers différents, et de fonctions permettant d'interagir avec ces objets. La classe RingModInstrument est la classe maître regroupant toutes les autres, et qui définit l'application en tant que tel (en utilisant les classes InputLine, RingMod, MidiServer, OutputLine et autres). 
Le fichier MESA_3_5.py est le point d'entrée de l'application : ce script instancie un objet RingModInstrument, ainsi que l'interface graphique permettant de contrôler l'instrument. Cette interface a été développée à l'aide de la librairie wxPython, et est essentiellement définie à l'intérieur du fichier GUI.py. Outre le traitement de signal, l'application possède également d'autres fonctionnalités utiles pour l'interprète : chaque paramètre graphique peut être mappé à un message MIDI, de telle sorte que l'on peut contrôler un paramètre en bougeant un potentiomètre sur une interface physique. Il est également possible de sauvegarder les valeurs des paramètres de l'application et de les charger à nouveau lors d'une utilisation future.

La première version de l'application, nommée simplement «MESA », a vu le jour en novembre 2016 et a connu huit versions successives, la dernière étant la version 3.5 (figure 6).

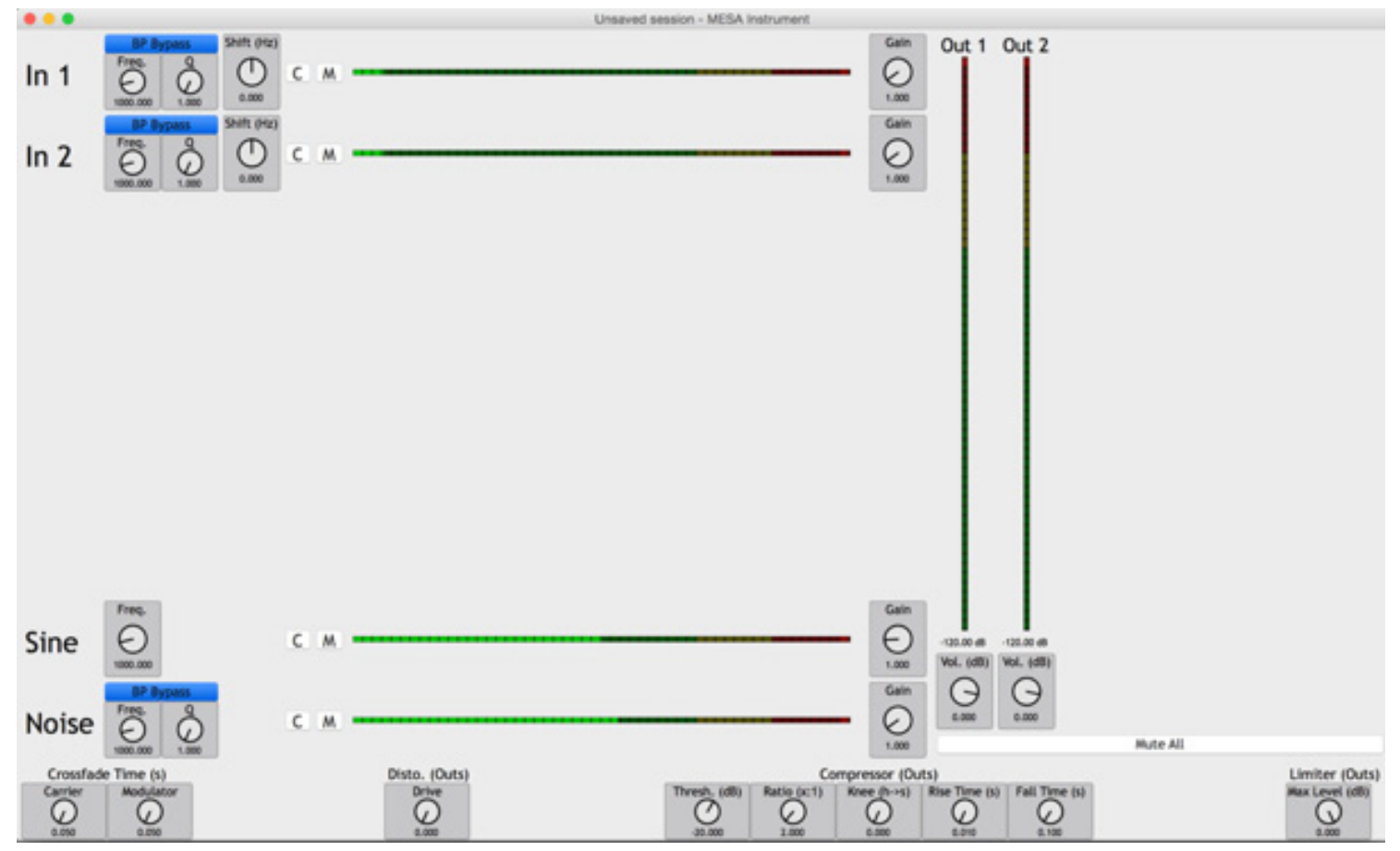

Figure 6: Capture d'écran du logiciel MESA v3.5 ; conception : Francis Lecavalier.

Malgré son souci d'authenticité, notons que Lecavalier a équipé son application de certains modules dont le dispositif originel n'était pas muni. Comme mentionné plus haut, une fonction de frequency shifter (translation de fréquence) a été ajoutée à l'entrée de chacun des signaux des bandonéons. Grâce à elle, le signal porteur était davantage inharmonique, permettant d'avoir accès à des sons à spectres largement variables, et ouvrant ainsi la porte à de nouvelles possibilités, sans pour autant changer le caractère du son original. Lecavalier a aussi agrémenté l'application d'un module de distorsion placé à la sortie du dispositif, configurable séparément sur chaque canal, dont le contrôle était laissé à la discrétion de l'interprète de la partie électronique (Pelz). Ce traitement permet de renforcer des partiels au signal entrant et à le rendre plus riche harmoniquement. La distorsion était effectuée par un objet Pyo Dist, qui combine l'application d'une fonction de transfert et d'un filtrage passe-haut sur le signal audio 
en entrée ${ }^{9}$. Le niveau de distorsion était paramétrable et servait à pallier la tendance du numérique d'être trop " propret » et de recréer quelque peu les aspérités du son typiques d'appareils analogiques des années 1960. En effet, comme c'est souvent le cas lorsqu'il est question de migration d'un univers analogique à un autre numérique, les failles et imprévisibilités inhérentes au traitement analogique du son se devaient aussi d'être modélisées.

Enfin, à la suite des suggestions de Pelz, Lecavalier a ajouté deux sources supplémentaires de modulation : en plus des quatre micros piézoélectriques, un générateur de bruit blanc et une onde sinusoïdale avec fréquence et amplitude paramétrables pouvaient également servir de sources pour le traitement de modulation. Ces deux signaux permettaient d'obtenir d'autres catégories de sonorités par rapport à l'emploi exclusif de signaux de bandonéon comme sons porteurs. Si ces modifications ne se justifient pas par rapport aux sources de documentation énumérées plus haut, elles nous semblaient s'inscrire néanmoins dans l'esprit expérimentaliste propre à Mumma et à son cercle. Bien que ces types de sources n'étaient pas présents dans le dispositif et dans l'œuvre originale en tant que telle, leur ajout pourrait se justifier par le fait que, selon le témoignage de Mumma, le compositeur utilisait couramment lui-même des générateurs d'ondes sinusoïdales ou des ondes triangulaires en tant qu'entrées pour tester son dispositif de $M e s a^{10}$. De ce point de vue, l'enrichissement des sources par des ondes sinusoïdales ou par du bruit blanc au sein du dispositif numérique s'inscrit dans les pratiques initiées par Mumma lui-même pour tester ses appareils, et n'est pas totalement étranger à celles-ci.

On ne saurait trop souligner l'apport de la communication directe avec le compositeur, même plus de 50 ans après la conception de l'œuvre. Cette correspondance servait parfois à valider certaines options de migration choisies; d'autres fois, elle avait au contraire l'effet de relativiser ou de nuancer les données recueillies par l'examen des documents d'archives. Le cas du statut du schéma électronique illustre bien ce cas de figure, car un chercheur qui ne travaillerait qu'avec la documentation sans interroger le compositeur aurait eu l'impression que le schéma constituait le plan définitif du dispositif de l'œuvre, alors que les échanges révélaient qu'il ne correspondait qu'à un plan général, et dont les modules spécifiques - ceux-là même responsables des différents traitements du son - étaient totalement absents. A contrario, si nous n'avions travaillé que sur la base des propos du compositeur, sans recours aux documents, notre lecture de l'œuvre aurait été incomplète et biaisée. C'est la jonction des deux ressources - le compositeur commentant post facto les documents génétiques produits à l'époque de la création - qui a fourni un portrait plus riche du processus de création de Mumma dans Mesa, et a ainsi permis le travail de récréation de l'œuvre. Ce modus operandi s'inspirait par ailleurs de la " génétique en direct »

9 Voir la documentation de l'objet Dist : http://ajaxsoundstudio.com/pyodoc/api/classes/effects.html\#pyo. Disto, consulté le 24 septembre 2019.

10 Voir plus haut le cinquième élément de l'énumération tirée d'un courriel de Mumma du 4 avril 2016, dans lequel il fait allusion à des «sine/square/triangle oscillators (for testing and balancing the sound levels without out the bandoneon (inputs, etc.)». 
que Nicolas Donin et Jacques Theureau ont proposée à travers une méthodologie connue sous le nom de "remise en situation ", une approche qu'ils ont appliquée à des études génétiques de compositeurs et d'interprètes tels que Philippe Leroux, Florence Baschet et Pierre-André Valade (voir par exemple, Donin 2010).

\section{PROBLÈMES D'INTERPRÉTATION}

Il restait à trouver une manière crédible d'interpréter la partie électronique de l'œuvre, tâche qui a été confiée au compositeur Ofer Pelz. Pelz a opté pour un contrôleur MIDI muni de plusieurs potentiomètres pour ajuster le niveau des différents paramètres mis en jeu dans la partie électronique, ainsi qu'un petit clavier MIDI pour contrôler la fréquence de l'onde sinusoïdale employée comme modulateur du son porteur dans certains passages de l'œuvre. Utiliser ces deux appareils externes à l'ordinateur portable permettait à Pelz d'apprendre des gestes instrumentaux à proprement parler et d'en acquérir une certaine maîtrise. Ce dispositif est semblable à ce qui s'utilise couramment dans le domaine de la musique mixte où des contrôleurs MIDI servent à intervenir sur des patchs dans Max/MSP ou Ableton Live.

Outre le rôle d'interprète de Pelz, qui était placé avec ses deux contrôleurs sur scène, vis-à-vis de l'instrumentiste Goldman, Lecavalier jouait également un rôle d'interprète ; positionné à la console de la régie, il se chargeait de la diffusion des quatre pistes de l'œuvre. Lors de la performance au festival MNM, Lecavalier mixait la pièce en direct en ajustant l'équilibre entre le son traité issu du dispositif à quatre canaux et le son non traité du bandonéon provenant des deux micros sur pied, pour un total de six canaux. Ce mixage permettait de faire varier les textures sonores de l'œuvre au cours de leurs évolutions, en ajustant les ratios son traité/son non traité, et d'augmenter la spatialisation du son à certains moments de la performance. Ce travail d'interprète hors scène a été réalisé dans un objectif de magnifier les sons et les mouvements spatiaux de la pièce.

Une autre dimension du nouveau dispositif proposé par Lecavalier résidait dans son intégration du hasard. Il s'agissait d'inventer un instrument qui imitait les aléas de l'analogique dans le cadre d'un algorithme parfaitement défini. Ce logiciel permettait à l'interprète d'intervenir sur plusieurs paramètres, notamment celui du timbre et de la spatialisation du son porteur du bandonéon. Comme les micros piézoélectriques reposaient directement sur l'instrument, ils vibraient en sympathie avec la caisse de résonance du bandonéon. Nos séances de travail ont permis à Pelz de comprendre quels microphones vibraient le plus en fonction des variations de paramètres que le logiciel permettait. Toutefois, même après plusieurs essais, le résultat conservait une part considérable d'aléatoire. Le travail en duo de Goldman et Pelz les a bien sûr conduits à réfléchir sur la forme de l'œuvre. Comme l'œuvre ne dispose pas de partition ni de schéma autre que le plan temporel excessivement dépouillé fourni par Mumma (figure 4), ils ont dû réfléchir à la façon dont ils allaient communiquer pendant l'exécution, et dont ils allaient construire ensemble une dramaturgie de l'œuvre. Certes, les deux musiciens se sont appuyés sur les deux enregistrements de l'œuvre réalisés par Mumma et Tudor, mais ils se sont vite rendu compte qu'ils devaient proposer d'autres articulations formelles afin de donner un sens à leur inter- 
prétation. D'une part parce que les deux enregistrements manifestent des caractéristiques sonores très différentes, mais aussi parce que le traitement s'entend dans les deux enregistrements bien plus que le son du bandonéon, de sorte qu'il s'avérait impossible de reconstituer les gestes instrumentaux du bandonéon de Tudor à l'aide de ces documents sonores. Même lorsqu'un de ces gestes était à peu près susceptible d'imitation au bandonéon, l'imiter machinalement semblait aller à l'encontre de l'essentielle ouverture de cette œuvre sans partition. S'inspirant des réalisations notées que Tudor a rédigées pour plusieurs des œuvres indéterminées de Cage, Goldman et Pelz ont décidé de dessiner un schéma graphique simple afin de les guider dans la forme de l'œuvre (figure 7).

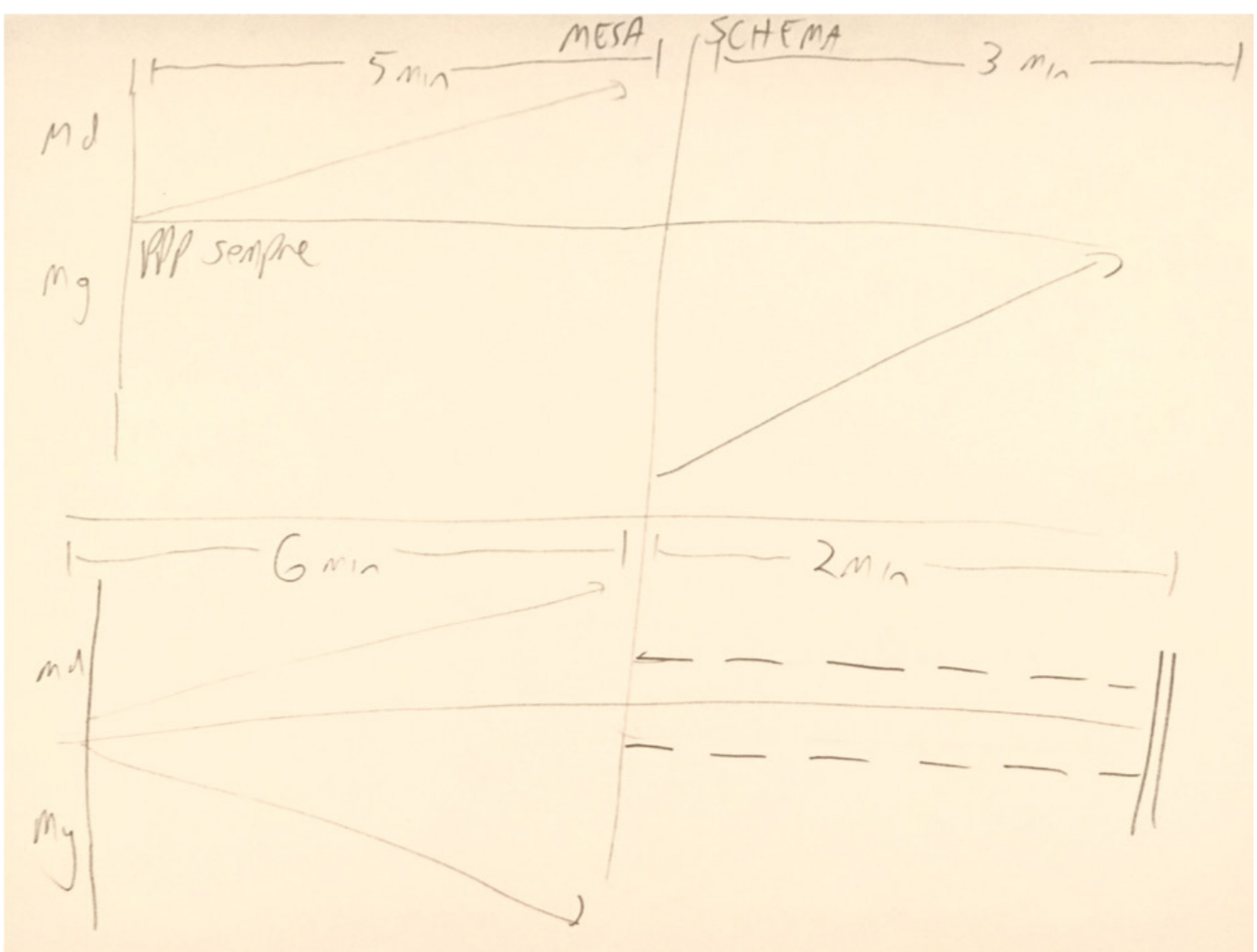

Figure 7 : Schéma graphique pour fins d'interprétation de Mesa, préparé par Jonathan Goldman et Ofer Pelz à l'automne 2016.

Ce schéma ad hoc constitue en effet une sorte de partition graphique calquée sur Mesa. La pièce y est divisée en quatre sections, chacune explorant des paramètres musicaux différents. Par exemple, une section constituée d'une longue montée à la main droite, une autre d'une lente montée à la main gauche, puis d'un long passage où la main droite monte pendant que la gauche descend vers le grave, l'œuvre se terminant par des clusters statiques aux deux mains ponctuées de pauses intermittentes. La durée fixée pour notre exécution étant de 16 minutes, les quatre segments duraient tour à tour cinq, trois, six et deux minutes, des durées assurées en concert par l'emploi d'un chronomètre partagé par Goldman et Pelz. Ce dernier se fiait au même 
schéma, même s'il n'apporte aucune information concernant le type de traitement à privilégier. Cette répartition était choisie afin d'éviter la perception d'une symétrie trop évidente dans la durée des différentes sections, tout en gardant la grande forme en deux sections à durée semblable (ici huit minutes par section) que Mumma avait définie à propos de la version studio de l'œuvre. En même temps, le schéma décrit des montées et descentes des deux mains du bandonéoniste qui ne sont pas inspirées des documents génétiques de l'œuvre ; elles ne sont là que pour permettre au musicien de remplir le temps avec intensité et concentration pendant l'exécution de l'œuvre, et ne s'entendent que peu à travers le traitement électronique. L'interprétation de Goldman, Pelz et Lecavalier a aussi été informée par une remarque faite par Mumma, évoquant la genèse de l'œuvre à partir de conversations qu'il eut avec Tudor lors du festival ONCE en 1965. Mumma a raconté que Tudor se disait ébloui par le long souffle du bandonéon, par le fait de pouvoir faire "chanter " une seule note pendant un très long laps de temps, et que le son produit soit doté d'une enveloppe très irrégulière. Mumma a affirmé que c'est à partir de ce principe du « long souffle » de l'instrument qu'il a conçu Mesa ; aussi notre interprétation contemporaine se devait de respecter cette préférence pour les notes tenues actionnées par un mouvement de soufflet irrégulier (Goldman 2008).

$$
* * *
$$

L'extrait audio suivant présente un aperçu de la nouvelle version de Mesa.

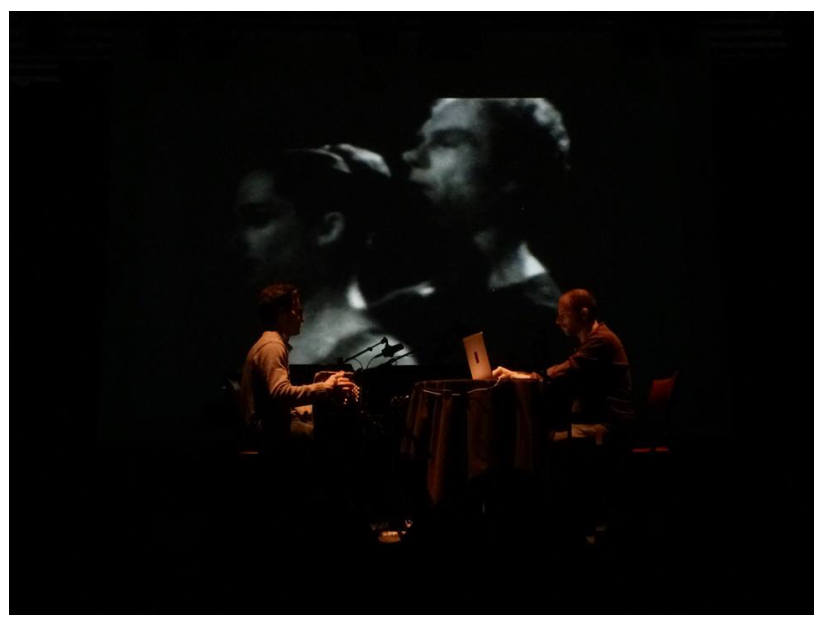

Extrait audio 3 : Extrait de Mesa interprétée par Jonathan Goldman, Francis Lecavalier et Ofer Pelz, version du 27 février 2017, Festival mNM, Agora Hydro-Québec, Montréal, avec projection d'extraits de deux captations filmées de la danse Place, montage de Preston Beebe (photo reproduite avec l'aimable autorisation de la sMce). Écouter l'extrait.

Si la version créée au terme de ce projet ne peut pas prétendre être identique - ni même isomorphique - à l'œuvre originale, le but était avant tout de mettre en scène les apories de l'œuvre indéterminée de façon générale. Non pas de substituer une indétermination par une détermination, mais plutôt de souligner le paradoxe (fécond) qui est le propre d'une œuvre à la fois absolument caractéristique, absolument impossible à confondre avec une autre, mais également tout à fait indéterminée, ses paramètres étant contingents, mobiles, ouverts à la négociation et issus du hasard ou des solutions pragmatiques à chaque exécution. Si nous sommes partis de l'hypothèse 
que l'œuvre se réduisait à son dispositif, force est d'admettre que l'objet étudié, Mesa, s'avère plus riche et plus ineffable que cette hypothèse ne le laissait entrevoir. En effet, l'œuvre dépasse finalement de beaucoup notre hypothèse initiale en ne se fondant pas complètement en son dispositif (poïétique) et en ne se réduisant pas à son incarnation sonore (esthésique) ; elle se situe plutôt entre ces deux pôles. De plus, l'importance de la correspondance avec le créateur démontre l'importance de la tradition "orale " (quoique transmise par courriel...) dans la transmission de l'œuvre - suggérant ainsi qu'aucun schéma de dispositif ne peut rendre compte complètement de l'œuvre. Néanmoins, Mesa témoigne d'une étonnante stabilité, assurée par les propriétés intrinsèques à la fois du bandonéon et du dispositif électronique : apprendre à jouer $\mathrm{du}$ bandonéon autrement afin d'être en phase avec le dispositif, tout en tentant, dans l'esprit de Cage, de soustraire sa propre volonté et son désir de produire telle ou telle réaction sonore, voilà ce qui constituait le défi central de l'œuvre, et (pourquoi pas ?) peut-être son essence aussi.

\section{BIBLIOGRAPHIE}

Baudouin, Olivier (2008), "Un exemple d'analyse à partir d'une partition codée. Stria de John Chowning ", communication présentée au colloque EMS'08, Paris, 7 juin, http://www.emsnetwork.org/ems08/abstract.html, consulté le 24 septembre 2019.

Bonardi, Alain (2013), « Pérenniser pour transmettre, transmettre pour pérenniser. Destins de l'œuvre mixte interactive : autour de En Echo de Philippe Manoury », Musique et technologie. Préserver, archiver, re-produire, Portraits polychromes, hors-série thématique, Paris, Institut National de l'audiovisuel, p. 105-126, https://hal.archives-ouvertes.fr/hal-01106912/document, consulté le 24 septembre 2019.

Bonardi, Alain, et Jérome Barthelemy (2008), " The Preservation, Emulation, Migration, and Virtualization of Live Electronics for Performing Arts. An Overview of Musical and Technical Issues ", Journal on Computing and Cultural Heritage, vol. 1, n ${ }^{\circ}$ 1, p. 6:1-16, https://dl.acm.org/citation.cfm?id=1367086, consulté le 21 août 2019.

Boutard, Guillaume, Catherine Guastavino, et James M. Turner (2013), " A Digital Archives Framework for the Preservation of Artistic Works with Technological Components ", The International Journal of Digital Curation, vol. 8, $\mathrm{n}^{\circ}$ 1, p. 42-65, https://www.semanticscholar. org/paper/A-Digital-Archives-Framework-for-the-Preservation-Boutard-Guastavino/4f4b875b38 a60a8c04de9384d99e523b6d0e6af7, consulté le 24 septembre 2019.

DeLio, Thomas (1993), «Structure as Context (1988)», dans Richard Kostelanetz (dir.), Writings about John Cage, Ann Arbor, University of Michigan Press, p. 163-175.

Donin, Nicolas (2010), "Quand l'étude génétique est contemporaine du processus de création: nouveaux objets, nouveaux problèmes ", Genesis, n ${ }^{\circ} 31$, p. 13-36, https://journals.openedition.org/ genesis/327, consulté le 24 septembre 2019.

Fillion, Michelle (2015), " Preamble. Gordon Mumma: A Short Biography », dans Gordon Mumma, Cybersonic Arts. Adventures in American New Music, Urbana, University of Illinois Press, p. xxvii-xxxiv.

Goldman, Jonathan (2008), Entretien avec Gordon Mumma, 25 octobre, Victoria, BC, Canada.

Goldman, Jonathan (2012), "The Buttons on Pandora's Box. David Tudor and the Bandoneon ", American Music, vol. 30, no 1 (printemps), p. 30-60, https://muse.jhu.edu/article/488556/pdf, consulté le 24 septembre 2019. 
Hartman, Lindsey Elizabeth (2019), « DIY in Early Live Electroacoustic Music. John Cage, Gordon Mumma, David Tudor, and the Migration of Live Electronics from the Studio to Performance ", these de doctorat, Louisiana State University, https://digitalcommons.lsu.edu/gradschool dissertations/4794/, consulté le 24 septembre 2019.

Hoffmann-Burchardi, Richard (2009), «Asymmetries Make the Difference. An Analysis of TransistorBased Analog Ring Modulators", Proceedings of the $12^{\text {th }}$ International Conference on Digital Audio Effects (DAFx-09), Como, Italy, 1-4 septembre, p. 1-4, http://dafx09.como.polimi.it/proceedings/ index.htm, consulté le 21 août 2019.

Johnson, Will (2011), "First Festival of Live-Electronic Music 1967 », dans Larry Austin et Douglas Kahn (dir.), Source. Music of the Avant-Garde 1966-1973, Berkeley/Los Angeles, University of California Press, p. 116-124.

Lemouton, Serge, et Samuel Goldszmidt (2016), « La préservation des œuvres du répertoire IRCAM. Présentation du modèle Sidney et analyse des dispositifs temps réel ", Actes des Journées d'Informatique musicale, 31 mars-2 avril, Albi, GMEA, p. 100-108, http://jim2016.gmea.net/actes, consulté le 21 août 2019.

Lombardo, Vincenzo, et al. (2009), « A Virtual-Reality Reconstruction of Poème Électronique Based on Philological Research ", Computer Music Journal, vol. 33, n 2, p. 24-47, https://www.jstor.org/ stable/40301026?seq=1\#metadata info tab contents, consulté le 24 septembre 2019.

Mumma, Gordon (1964) «An Electronic Music Studio for the Independent Composer », Journal of the Audio Engineering Society, vol. 12, no 3, p. 240-244 ; rééd. dans Circuit, musiques contemporaines, vol. $19 \mathrm{n}^{\circ}$ 3, 2009, p. 85-90, https://www.erudit.org/en/journals/circuit/2009-v19-n3circuit3473/038261ar.pdf, consulté le 24 septembre 2019 ; rééd. dans Mumma 2015, p. 14-22.

Mumma, Gordon (2015), Cybersonic Arts. Adventures in American New Music, édité et commenté par Michelle Fillion, Champaign, IL, University of Illinois Press.

Pottier, Laurent (2013), «La régénération des sons de Turenas de John Chowning», dans Evelyne Gayou (dir.), Préserver - Archiver - Re-produire. Musique et technologie, jeux vidéo, Portraits polychromes, horssérie thématique, Paris, Ina-GRM, p. 145-196.

Wetzel, David Brooke (2006), «A Model for the Conservation of Interactive Electroacoustic Repertoire. Analysis, Reconstruction, and Performance in the Face of Technological Obsolescence », Organised Sound, vol. 11, no 3 (décembre), p. 273-284, https://doi.org/10.1017/S1355771806001555, consulté le 24 septembre 2019.

Zattra, Laura (2007), "The Assembling of Stria by John Chowning. A Philological Investigation », Computer Music Journal, vol. 31, no 3, p. 38-64, https://www.jstor.org/stable/40072593, consulté le 24 septembre 2019.

\section{DisCOGRAPHIE}

Kagel, Mauricio, Gordon Mumma, et Christian Wolff (1968), A Second Wind for Organ, David Tudor (orgue, bandoneon) et Gordon Mumma (électronique), disque 33 tours, Odyssey 32160158.

Mumma, Gordon (2002), Gordon Mumma. Live Electronic Music, Tzadik Records TZ 7074.

Wolff, Christian, et al. (2010), Music for Merce (1952-2009), coffret de 10 CD, New World Records 807122 
Annexe : Chronologie des Échanges avec Gordon Mumma et conception du disPositif

31 mai 2016: Création du premierschéma del'application MESA par Francis Lecavalier (" MESA, draft \#1 »), principalement à partir de deux sources : l'article de Jonathan Goldman (2012, p. 46-50), mais surtout grâce un message de Mumma envoyé à Jonathan et datant du 5 avril 2016 («MESA (1966), brief tech details»).

Ce message contient une description sommaire, mais relativement complète $\mathrm{du}$ traitement audio utilisé dans la pièce et des principaux composants utilisés (modulateurs en anneau, filtres passe-bande, suiveurs d'enveloppe, etc.).

Le schéma électronique classique de MESA, ("MESA, two source duo ») n'a pas été utile, puisque Lecavalier n'avait pas les connaissances techniques requises pour le lire.

Début juin 2016 : Envoi du premier schéma de $M E S A$ à Mumma.

3 juin 2016 : Réponse initiale de Mumma, qui indique qu'il fouillera dans ses archives de Mesa vers la mi-juin pour comparer avec le premier schéma de Lecavalier.

5 juillet 2016: Courriel de Mumma détaillant le processus technique et le raisonnement derrière Mesa, à partir de ses notes personnelles datant des années 1960. Il y explique sa réflexion derrière l'utilisation des modulateurs en anneaux (" ring modulators"), détaille un peu plus l'utilisation des micros piézoélectriques et des " micros aériens " (" air mics ») et décrit aussi rapidement la manière dont il " routait " les différents signaux entre eux.

Le courriel ne fait pas vraiment mention du premier brouillon de Lecavalier. Il ne fournit pas non plus beaucoup de nouvelles informations, mais apporte surtout un contexte historique et davantage de détails sur certains éléments du dispositif.

Cependant, c'est notamment grâce à ce courriel que nous avons appris qu'il y avait quatre micros piézoélectriques (deux de chaque côté). Nous pensions auparavant qu'il n'y en avait que deux.

19 juillet 2016 : Création du deuxième schéma de MESA par Lecavalier, («MESA draft \#2 ") à partir des précisions de Mumma dans son dernier courriel. La différence principale réside dans l'ajout de deux micros piézoélectriques supplémentaires, pour un total de quatre (contrairement au schéma \#1 qui n'en comportait que deux). Il ajoute également les deux "air mics ». Lecavalier a supprimé la boucle de feedback du modulateur en anneau qui était présente dans le premier schéma : comme le compositeur n'a jamais mentionné ce renvoi des sorties vers les entrées dans ses notes, Lecavalier en a conclu que le renvoi n'avait pas lieu d'être.

Nous ne sommes pas certains que ce deuxième schéma ait été transmis à Mumma : si c'est le cas, sa réponse n'a pas été conservée.

23 septembre 2016 : Création du «Schéma de connexions physiques » du dispositif par Lecavalier. Ce schéma détaille le parcours du signal audio à travers le dispositif d'une manière plus " macro ». Nous sommes au niveau des connexions physiques à effectuer entre les différents appareils (ordinateur, micros, console de son, etc.) pour la performance. 
25 octobre 2016 : Envoi de la toute première version du logiciel Python à Ofer Pelz, MESA 1.0.

26 octobre 2016 : Mumma donne sa bénédiction à Goldman, Pelz et Lecavalier pour faire le concert du 27 février 2017 sans lui. Le même jour, il envoie un courriel à Goldman avec une carte très sommaire de la structure de Mesa ("General structural map of MESA (1966)»).

31 octobre 2016 : Envoi de $M E S A 1.1$ à Pelz (2 version du logiciel).

8 novembre 2016 : Envoie de MESA 1.2 à Pelz (3e version du logiciel).

12 novembre 2016 : Envoi de $M E S A 2.0$ à Pelz (4 $4^{\mathrm{e}}$ version du logiciel)

23 novembre 2016 : Mumma envoie un courriel à Goldman apportant des détails sur la durée de la pièce (très variable, entre 18 et 24 minutes).

20 décembre 2016 : Envoi de $M E S A 3.0$ (version incomplète) à Pelz ( $5^{\mathrm{e}}$ version du logiciel).

13 janvier 2017 : Envoi de MESA 3.0 (version complète) à Pelz (6 $6^{\mathrm{e}}$ version du logiciel).

20 janvier 2017 : Envoi de $M E S A 3.1$ à Pelz (7e version du logiciel).

25 février 2017 : Envoi de $M E S A 3.5$ à Pelz (8 et dernière version). 